\title{
Meson spectroscopy at VES and COMPASS
}

\author{
Dmitry Ryabchikov ${ }^{1,2, *}$ \\ for the VES group and the COMPASS collaboration \\ ${ }^{1}$ NRC Kurchatov Institute, IHEP, Protvino, Russia \\ ${ }^{2}$ Technical University of Muenchen, Garching, Germany
}

\begin{abstract}
Diffractive production of $\pi^{-} \pi^{-} \pi^{+}$and $\pi^{-} \pi^{0} \pi^{0}$ final states is the subject of comprehensive studies performed recently by the VES and the COMPASS experiments. COMPASS pioneered the application of novel methods of partial-wave analysis: mass-independent PWA in multiple $\left(\boldsymbol{m}_{3 \pi}, \boldsymbol{t}^{\prime}\right)$-cells, massdependent analysis of spin-density matrices performed simultaneously in all measured $\boldsymbol{t}^{\prime}$ bins, the analysis with freed shapes of $\pi^{+} \pi^{-}$isobars. In addition, COMPASS observed a new narrow state: $\boldsymbol{a}_{\mathbf{1}}(\mathbf{1 4 2 0})$. VES has world-leading data samples on $\boldsymbol{\pi}^{-} \boldsymbol{\pi}^{-} \boldsymbol{\pi}^{+}$and $\boldsymbol{\pi}^{-} \boldsymbol{\pi}^{0} \boldsymbol{\pi}^{0}$, that yield compatible results and show the potential for a detailed comparison of isospin relations between different decay channels, using the PWA methods with fixed and freed shapes of $\pi \pi$ isobars.
\end{abstract}

\section{Introduction}

The diffractive production of $3 \pi$ states is currently the subject of detailed studies by the VES [1] and the COMPASS [2] experiments. Both experiments perform an exclusive measurement of the reaction $\pi_{\text {beam }}^{-} N_{\text {target }} \rightarrow 3 \pi N_{\text {recoil }}$, where $N$ is a nuclear or proton target. The VES experiment runs at a beam momentum of $29 \mathrm{GeV} / c$, uses a Be target, and covers the squared four-momentum interval $0<t^{\prime}<1.0(\mathrm{GeV} / \mathrm{c})^{2}$. COMPASS uses a $190 \mathrm{GeV} / \mathrm{c}$ beam on a proton target and covers the range $0.1<t^{\prime}<1.0(\mathrm{GeV} / c)^{2}$.

The numbers of events, selected for the presented analysis, are $87 \times 10^{6} \pi^{-} \pi^{-} \pi^{+}$and $32 \times 10^{6} \pi^{-} \pi^{0} \pi^{0}$ for VES and $46 \times 10^{6} \pi^{-} \pi^{-} \pi^{+}$and $3.5 \times 10^{6} \pi^{-} \pi^{0} \pi^{0}$ for COMPASS.

The latest results of the VES analysis of the $\pi^{-} \pi^{-} \pi^{+}$and $\pi^{-} \pi^{0} \pi^{0}$ were presented in [3-8]. VES, having compatible statistics of $\pi^{-} \pi^{-} \pi^{+}$and $\pi^{-} \pi^{0} \pi^{0}$, presents results for both systems $[3,4]$. VES reported preliminary results on a resonance-model fit of the $\pi^{-} \pi^{0} \pi^{0}$ data $[5,8]$ and a partial-wave analysis (PWA) with freed $\pi^{0} \pi^{0} S$-wave isobar amplitudes of the $\pi^{-} \pi^{0} \pi^{0}$ data [8].

The COMPASS results for the $\pi^{-} \pi^{-} \pi^{+}$final states were published in [9-11]. Results on $\pi^{-} \pi^{0} \pi^{0}$ are presented in $[12,13]$. COMPASS concentrates on the analysis of $\pi^{-} \pi^{-} \pi^{+}$ states, and has performed a detailed PWA with 88 waves (fixed isobars), a PWA with freed $\pi^{+} \pi^{-} S$-wave isobar amplitudes [10], and a resonance-model fit of 14 selected waves that are described simultaneously in $11 t^{\prime}$ bins using 11 isovector resonances [9].

The short-hand notation to define $3 \pi$ partial waves is

$$
J^{P C} M^{\varepsilon} r \pi L,
$$

where $J^{P C} M^{\varepsilon}$ are the quantum numbers of the $3 \pi$ system, $r$ is the $\pi \pi$ isobar and $L$ is the relative orbital angular momentum between isobar $r$ and the bachelor pion $\pi$.

\footnotetext{
*e-mail: Dmitry.Ryabchikov@ihep.ru
} 


\section{Comparison of $\pi^{-} \pi^{-} \pi^{+}$and $\pi^{-} \pi^{0} \pi^{0}$ final states}

The simultaneous analysis and comparison of $\pi^{-} \pi^{-} \pi^{+}$and $\pi^{-} \pi^{0} \pi^{0}$ final states is important because in the isobar model, isospin symmetry relates the intensities and relative phases in the two systems.

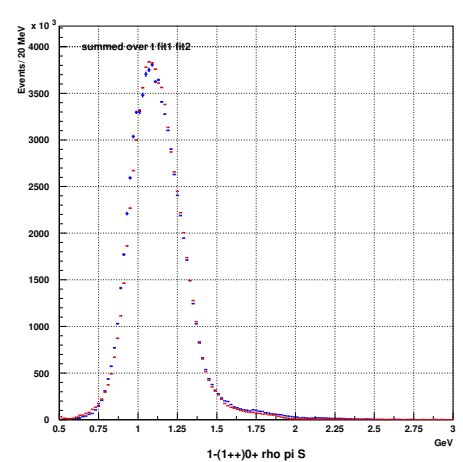

(a)

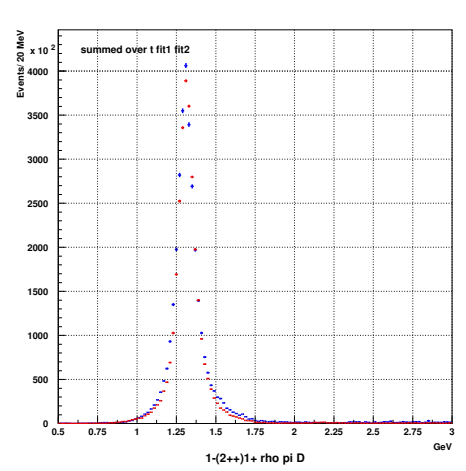

(c)

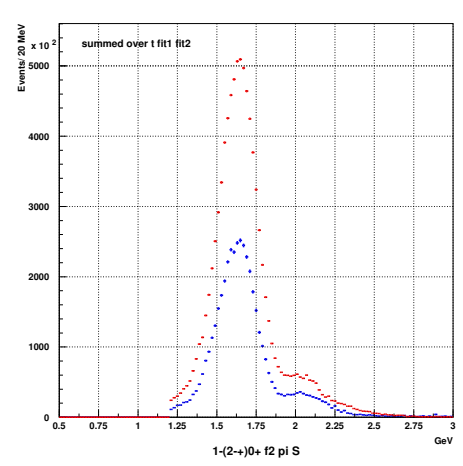

(e)

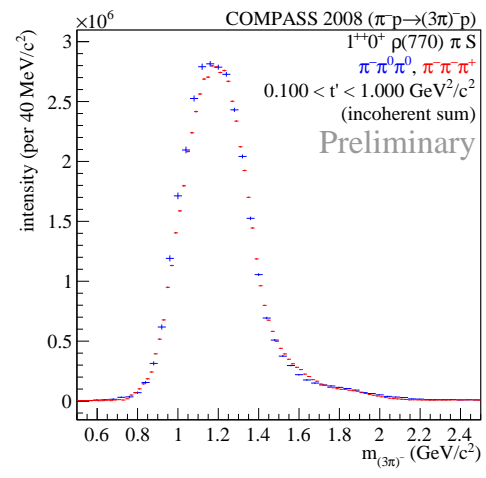

(b)

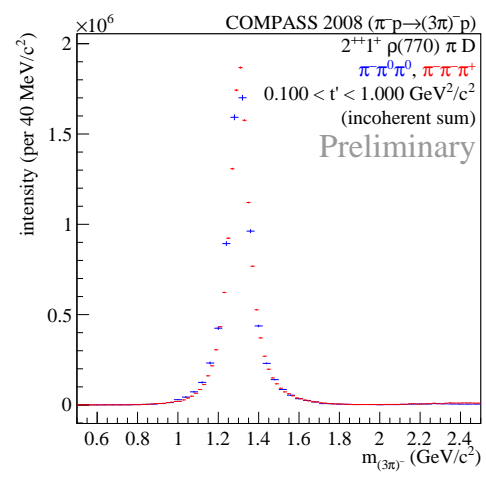

(d)

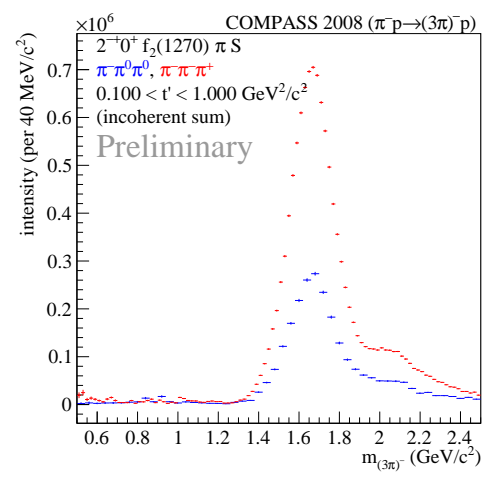

(f)

Figure 1: $1^{++} 0^{+} \rho(770) \pi S$ intensity: VES (a), COMPASS (b). $2^{++} 1^{+} \rho(770) \pi D$ intensity: VES (c), COMPASS (d). $2^{-+} 0^{+} f_{2}(1270) \pi S$ intensity: VES (e), COMPASS (f). The $\pi^{-} \pi^{-} \pi^{+}$ data are shown in red, the $\pi^{-} \pi^{0} \pi^{0}$ data in blue.

Waves with isovector $\pi \pi$ isobars (e.g. $\rho(770) \pi$ waves) are expected to be equal between the neutral and the charged decay modes, i.e. $\operatorname{BR}\left(X^{-} \rightarrow \pi^{-} \pi^{0} \pi^{0}\right) / \operatorname{BR}\left(X^{-} \rightarrow \pi^{-} \pi^{-} \pi^{+}\right)=1$ 
if we do not consider $I>1$. Waves with isoscalar $\pi \pi$ isobars (e.g. $f_{2}(1270) \pi$ or $f_{0}(980) \pi$ waves) in the $\pi^{-} \pi^{0} \pi^{0}$ are expected to be half of the intensity in the $\pi^{-} \pi^{-} \pi^{+}$because $\mathrm{BR}\left(f \rightarrow \pi^{0} \pi^{0}\right) / \mathrm{BR}\left(f \rightarrow \pi^{-} \pi^{+}\right)=1 / 2$. However, taking into the account self-interferrence due to bose symmetrization in $\pi^{-} \pi^{-} \pi^{+}$, the value $\operatorname{BR}\left(X^{-} \rightarrow \pi^{-} \pi^{0} \pi^{0}\right) / \operatorname{BR}\left(X^{-} \rightarrow \pi^{-} \pi^{-} \pi^{+}\right)$can be far away from $1 / 2$. This happens in the case of broad $\pi \pi$ isobars such as $f_{0}(600)$.

Both experiments use similar approaches to analyze the data. The data are divided into set of equidistant $m_{3 \pi}$ bins and non-equidistant $t^{\prime}$ bins (so-called $t^{\prime}$-resolved analysis). The partial-wave analysis is applied independently in each $\left(m_{3 \pi}, t^{\prime}\right)$ bin, using a rank-1 spin-density matrix for dominant $\varepsilon=+1$ amplitudes. The angles of the outgoing pions are defined such that the relative phases in $\pi^{-} \pi^{-} \pi^{+}$and $\pi^{-} \pi^{0} \pi^{0}$ are the same.

Figure 1 shows the intensities of three dominant partial waves in $m_{3 \pi}$ bins, summed over $t^{\prime}$. The $1^{++} 0^{+} \rho(770) \pi S$ intensities from VES (figure 1(a)) and COMPASS (figure 1(b)) exhibit a broad bump that appears at lower $m_{3 \pi}$ in the VES data. This points to complicated composition of the $1^{++}$wave, explained by $a_{1}(1260)$ resonance signal and significant amount of coherent non-resonant background in the resonance-model fit of the COMPASS data [9]. The $2^{++} 1^{+} \rho(770) \pi D$ intensity is shown in figure 1 (c) for VES and in figure $1(\mathrm{~d})$ for COMPASS. It shows clear peak of the $a_{2}(1320)$ meson. Both experiments find the expected 1:1 intensity ratio for the $\rho \pi$ decay into $\pi^{-} \pi^{-} \pi^{+}$(red) and $\pi^{-} \pi^{0} \pi^{0}$ (blue). Figures 1(e) and 1(f) show the $2^{-+} 0^{+} f_{2}(1270) \pi S$ intensity for VES and COMPASS, respectively, which is dominated by the $\pi_{2}(1670)$ resonance. In this case, the intensity ratio of $\pi^{-} \pi^{0} \pi^{0}$ to $\pi^{-} \pi^{-} \pi^{+}$is close to $1 / 2$ at $1.7 \mathrm{GeV} / c^{2}$, which is in agreement with the expectation from the isobar model.

The phases of the $2^{++}$and $2^{-+}$waves relative to the $1^{++}$wave (not shown here) demonstrate a striking similarity for two experiments and for two final states. The phases also have only a weak dependence on $t^{\prime}$. This points to the observation of the same production mechanism for the dominant resonances, even at the lower beam energies of VES, where the Reggeon exchange (for instance $\rho$ exchange) could be significant in addition to Pomeron exchange.

\section{The $a_{1}(1420)$}

A novel resonance-like signal, the $a_{1}(1420)$, that appears as a narrow peak in the $1^{++} 0^{+}$ $f_{0}(980) \pi P$ wave at about $1.4 \mathrm{GeV} / c^{2}$, and that is associated with a rapid phase rise was first discovered in the $\pi^{-} \pi^{-} \pi^{+}$COMPASS data [11]. Resonance-model fits using 3 waves [11] and 14 waves [9] were performed, the latter yields the parameters: $m_{a_{1}(1420)}=1411_{-5}^{+4} \mathrm{MeV} / c^{2}$ and $\Gamma_{a_{1}(1420)}=161_{-14}^{+11} \mathrm{MeV} / c^{2}$, where uncertainties are dominated by systematics. COMPASS also observes the $a_{1}(1420)$ peak without modeling the line shape of the $f_{0}(980)$ using the freed-isobar approach [10]. VES has reported preliminary results of a resonance-model fit of the $1^{++} 0^{+} f_{0}(980) \pi P$ wave using subset of the $\pi^{-} \pi^{0} \pi^{0}$ data [8], which are consistent with COMPASS ( $m_{a_{1}(1420)}$ is $10 \mathrm{MeV} / c^{2}$ higher compared to COMPASS). The nature of the $a_{1}(1420)$ is highly disputed, so this phenomena needs further investigation, using different data sets.

Figures 2(a) and 2(b) show $1^{++} 0^{+} f_{0}(980) \pi P$ intensity distribution for VES and COMPASS, respectively (in the latter $\pi^{-} \pi^{-} \pi^{+}$is scaled to $\pi^{-} \pi^{0} \pi^{0}$ ). Figures 2(c) and 2(d) show for the lowest $t^{\prime}$ bin the relative phase between $1^{++} 0^{+} f_{0}(980) \pi P$ and $1^{++} 0^{+} \rho(770) \pi S$. Both intensities and relative phases look similar above $m_{3 \pi}=1.4 \mathrm{GeV} / c^{2}$ for the two experiments. The differences at low masses could be explained by different background conditions, which can depend on beam energy, $t^{\prime}$ region and target. The current VES data, demonstrating accurate isospin relation in the peak of $a_{1}(1420)$ and having small statistical uncertainties in both $\pi^{-} \pi^{-} \pi^{+}$and $\pi^{-} \pi^{0} \pi^{0}$ modes, looks very promising for further more detailed analysis, including freed-isobar methods. 


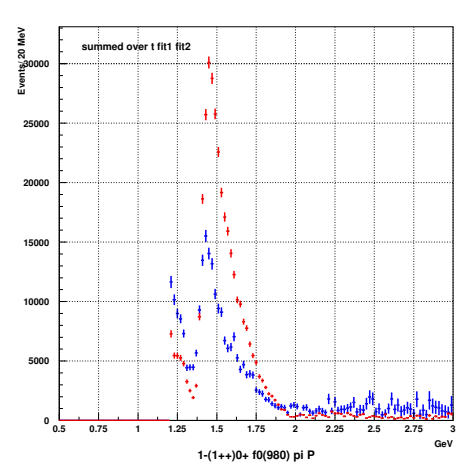

(a)

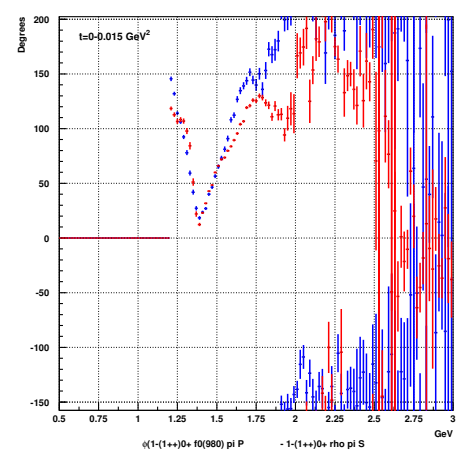

(c)

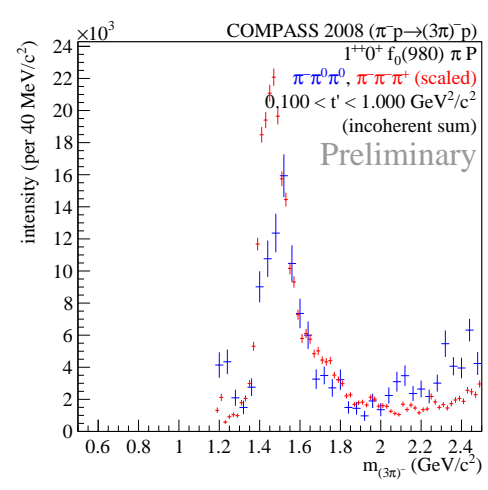

(b)

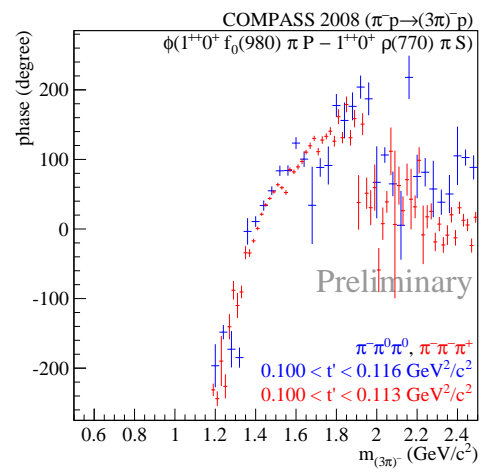

(d)

Figure 2: $1^{++} 0^{+} f_{0}(980) \pi P$ intensity VES (a), COMPASS (b). Relative phase between the $1^{++} 0^{+} f_{0}(980) \pi P$ and the $1^{++} 0^{+} \rho(770) \pi S$ VES (c), COMPASS (d). The $\pi^{-} \pi^{-} \pi^{+}$data are shown in red, the $\pi^{-} \pi^{0} \pi^{0}$ data in blue.

\section{Freed-isobar PWA}

The freed-isobar PWA is a novel approach so the method is briefly introduced. In the conventional PWA of $3 \pi$ final states, the decay amplitudes (partial waves) are complex functions $\Psi_{i}(\tau)$ that are labeled by quantum numbers $i$, as is given by Eq.(1), and depending on the $3 \pi$ phase-space variables $\tau$ :

$$
\Psi_{i}(\tau)=\sum_{k=1}^{N_{\mathrm{perm}}} \mathcal{D}_{r}\left(m_{r, k}\right) F_{J_{r}}\left(m_{r, k}\right) \mathcal{K}_{i}\left(\Omega_{k}^{\mathrm{GJ}}, \Omega_{k}^{\mathrm{HF}}\right) .
$$

They contain functions $\mathcal{K}_{i}\left(\Omega_{k}^{\mathrm{GJ}}, \Omega_{k}^{\mathrm{HF}}\right)$ that collect all angular dependencies and depend on the angles in Gottfried-Jackson and helicity rest frames, centrifugal-barrier factors $F_{J_{r}}\left(m_{r}\right)$ and the amplitude $\mathcal{D}_{r}\left(m_{r}\right)$ describing the propagation of $\pi \pi$ isobar. Summing over index $k$ represents the Bose symmetrization, where the kinematic variables are calculated for different combinations of the final-state pions.

In the freed-isobar method, the amplitude $\mathcal{D}\left(m_{r}\right)$ is presented by a set of piece-wise constant functions $\Pi_{l, r}\left(m_{r}\right)$ that fully cover the allowed mass range for $m_{r}$, multiplied on complex coefficients $T_{l}$ :

$$
\mathcal{D}\left(m_{r}\right)=\sum_{l}^{N_{\text {bins }}} T_{l} \Pi_{l, r}\left(m_{r}\right), \quad \Pi_{l, r}\left(m_{r}\right)= \begin{cases}1 & \text { if } m_{r, l} \leq m_{r}<m_{r, l+1}, \\ 0 & \text { otherwise }\end{cases}
$$


By substituting Eq.(3) into Eq.(2) and taking out $T_{l}$ (used as fitting parameters), we construct a new set of decay amplitudes:

$$
\widetilde{\Psi}_{i, l}(\tau)=\sum_{k=1}^{N_{\text {perm }}} \Pi_{l, r}\left(m_{r, k}\right) F_{J_{r}}\left(m_{r, k}\right) \mathcal{K}_{i}^{\varepsilon}\left(\Omega_{k}^{\mathrm{GJ}}, \Omega_{k}^{\mathrm{HF}}\right) .
$$

In the reflectivity basis, the amplitudes (4) are real-valued functions, without any free parameters, so the standard PWA technique can be used. Using $T_{i, l}$ as fit parameters and wave-set Eq.(4), the over-all strength of the partial wave with quantum numbers $i$ and the isobar amplitude $\mathcal{D}\left(m_{r}\right)$ will be optimized.

It was found that the freed wave sets of the form of Eq. (4) contain linear-dependencies, which are exact in the limit of zero $m_{r}$ bin width for $\Pi_{l, r}\left(m_{r}\right)$. Sets of functions were found, keeping the total $3 \pi$ amplitude unchanged, when added to each $[\pi \pi]_{j}$ isobar amplitude. Such functions are called zero modes (ZM) [14]. These linear dependencies arise only in case of Bose-symmetrization in $3 \pi$, which breaks the orthogonality between angular amplitudes in Eq.(4). They appear when different values of $L$ and spin $j$ of the $\pi \pi$ isobar are used for the same $J^{P C} M^{\varepsilon}$. The case when the one amplitude has one $\mathrm{ZM}$, is $1^{-+} 1^{+}[\pi \pi]_{P} \pi P$. $\mathrm{ZM}$ is constant function of $m_{r}$.

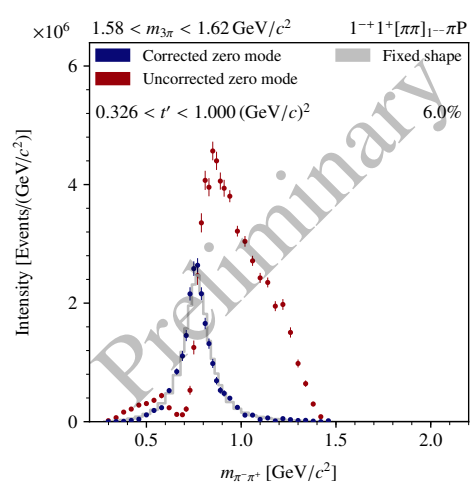

(a)

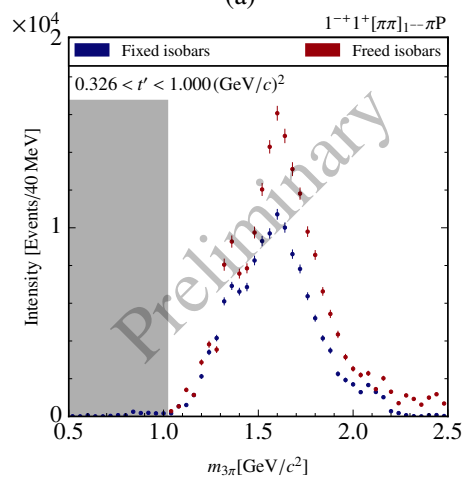

(c)

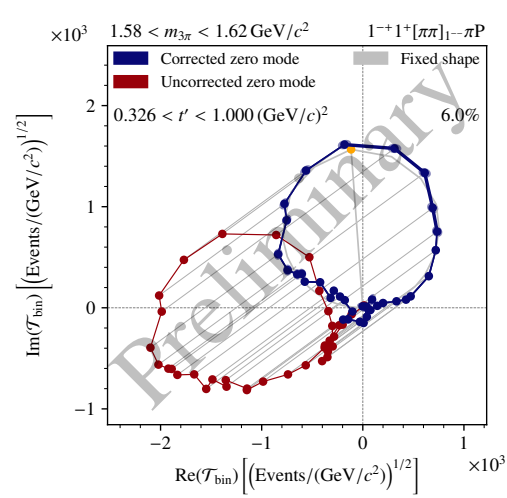

(b)

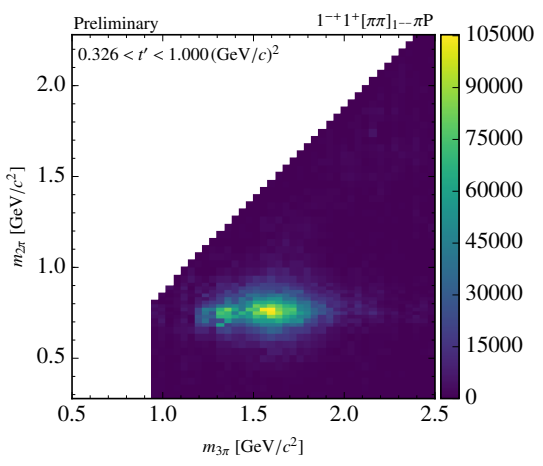

(d)

Figure 3: Intensity distribution (a) and Argand diagram (b) for the freed $[\pi \pi]_{P}$ amplitude before (red) and after (blue) fixing the zero mode. The $1^{-+} 1^{+}[\pi \pi]_{P} \pi P$ intensity in $m_{3 \pi}$ bins (c) from the freed-isobar PWA (red) and the fixed-isobar PWA (blue). Intensity of $1^{-+}$as a function of $m_{3 \pi}$ and $m_{2 \pi}$ after fixing the zero mode (d). 
The latest freed-isobar PWA of the COMPASS data uses 12 waves with freed isobar amplitudes, including the spin-exotic $1^{-+} 1^{+}[\pi \pi]_{P} \pi P$ wave [15]. Figures $3(\mathrm{a})$ and $3(\mathrm{~b})$ show the intensity and Argand diagram for freed $[\pi \pi]_{P}$ amplitude in $1^{-+}$, obtained in a single $\left(m_{3 \pi}, t^{\prime}\right)$ bin. The obtained PWA solution is shown in red, which looks very different from the expected $\rho(770)$ resonance line shape. Due to the ZM ambiguity, each data point has infinite uncertainties. Therefore, the error-bars were modified by removing the eigenvector along the $\mathrm{ZM}$ direction from the covariance matrix. To resolve the ambiguity, the complex amplitude of $[\pi \pi]_{P}$ was required to be as close as possible to Breit-Wigner amplitude of $\rho(770)$ for $m_{\pi^{-} \pi^{+}}<1.12 \mathrm{GeV} / c^{2}$, which requires fit with one complex parameter. The result of this fit is shown in figures 3(a) and 3(b) in blue and the Breit-Wigner curves are shown in gray, demonstrating nice agreement with $\rho(770)$. Figure 3(c) shows $1^{-+}$intensity in all $m_{3 \pi}$ bins obtained from the freed isobar fit in red, and the one from the fixed-isobar PWA in blue. Figure 3(d) shows the intensity of $1^{-+}$as function of $m_{3 \pi}$ and $m_{2 \pi}$, after the ZM ambiguity is resolved in all $m_{3 \pi}$ bins. The plot shows a clear peak at $m_{3 \pi}=1.6 \mathrm{GeV} / c^{2}$ and $m_{2 \pi}=0.77$ $\mathrm{GeV} / c^{2}$, corresponding to decay $\pi_{1}^{-}(1600) \rightarrow \rho(770) \pi^{-}$. This shows that $\pi_{1}^{-}(1600)$ found in the conventional analysis [9] is not an artifact due to using fixed shapes of isobars.

\section{Conclusions and Outlook}

We compare for the first time the mass-independent PWA results for the $\pi^{-} \pi^{-} \pi^{+}$and $\pi^{-} \pi^{0} \pi^{0}$ final states that were obtained by the VES and COMPASS experiments. For both experiments, the intensities of 3 dominant partial waves exhibit the isospin relations between $\pi^{-} \pi^{-} \pi^{+}$and $\pi^{-} \pi^{0} \pi^{0}$, that are expected for the isobar model. The $1^{++} 0^{+} f_{0}(980) \pi P$ wave shows narrow peak in its intensity and a rapid phase rise near $1.4 \mathrm{GeV} / c^{2}$, attributed to the $a_{1}(1420)$. The VES experiment demonstrates the potential for detailed study of $a_{1}(1420)$ in both $\pi^{-} \pi^{-} \pi^{+}$ and $\pi^{-} \pi^{0} \pi^{0}$ final states. The extended freed-isobar fits of COMPASS $\pi^{-} \pi^{-} \pi^{+}$data show the possibility of deep insight into the spin-exotic $1^{-+} 1^{+} \rho(770) \pi P$ wave and the $\pi_{1}(1600)$.

\section{Acknowledgments}

This work was supported by RFBR grant 16-02-00737, the DFG Collaborative Research Centre/Transregio 110 and the Excellence Cluster 'Universe'.

\section{References}

[1] Yu. Khokhlov et al., EPJ Web Conf. 37, 01029 (2012)

[2] P. Abbon et al. (COMPASS), Nucl. Instrum. Meth. A779, 69 (2015)

[3] I. Kachaev, D. Ryabchikov, EPJ Web Conf. 199, 02025 (2019)

[4] I. Kachaev, D. Ryabchikov, EPJ Web Conf. 130, 04003 (2016)

[5] D. Ryabchikov et al., AIP Conf. Proc. 1701, 040020 (2016)

[6] I. Kachaev et al., Phys. Atom. Nucl. 78, 1474 (2015)

[7] I. Kachaev et al., in Proceedings, 20th International Conference on Particles and Nuclei (PANIC 14): Hamburg, Germany, August 24-29, 2014 (2014), pp. 185-189

[8] Yu. Khokhlov et al., PoS Hadron2013, 088 (2013)

[9] M. Aghasyan et al. (COMPASS Collaboration), Phys. Rev. D 98, 092003 (2018)

[10] C. Adolph et al. (COMPASS Collaboration), Phys. Rev. D95, 032004 (2017)

[11] C. Adolph et al. (COMPASS Collaboration), Phys. Rev. Lett. 115, 082001 (2015)

[12] S. Uhl (COMPASS), PoS Hadron2013, 087 (2013)

[13] S. Uhl (COMPASS), EPJ Web Conf. 164, 07045 (2017)

[14] F. Krinner, D. Greenwald, D. Ryabchikov, B. Grube, S. Paul, Phys.Rev.D 97, 114008 (2018)

[15] F. Krinner (COMPASS), EPJ Web Conf. 199, 02003 (2019) 